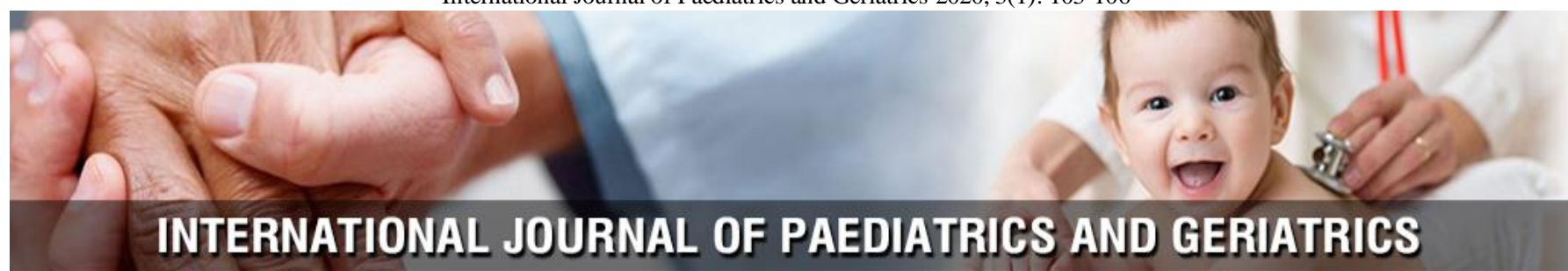

P-ISSN: 2664-3685

E-ISSN: 2664-3693

www.paediatricjournal.com

IJPG 2020; 3(1): 103-106

Received: 17-11-2019

Accepted: 18-12-2019

Dr. Paka Rajanna Rajender Associate Professor, Department of Paediatrics, RVM Institute of Medical Sciences, Siddipet, Telangana, India

Dr. Manoj Patruni Assistant Professor, Department of Community Medicine RVM Institute of Medical Sciences, Siddipet, Telangana, India
Corresponding Author: Dr. Manoj Patruni Assistant Professor, Department of Community Medicine RVM Institute of Medical Sciences, Siddipet, Telangana, India

\section{Assess the effectiveness of oral versus parenteral antibiotics in children under five years of age, diagnosed with severe pneumonia in a private teaching hospital, Telangana state}

\author{
Dr. Paka Rajanna Rajender and Dr. Manoj Patruni
}

DOI: https://doi.org/10.33545/26643685..v.i.68

\begin{abstract}
Introduction: The most significant and striking feature of pneumonia is consolidation. Pneumonia continues to be the biggest killer disease globally, of less than five year's children. Pneumonia accounts for $15 \%$ of all deaths of children under 5 years old, killing 8, 08694 children in 2017.

Methodology: Hospital based prospective observational comparative study. This study was conducted in the department of paediatrics, RVM institute of medical sciences and research canter. This study was approved by the RVM institutional ethical committee prior to the study. The study was conducted between the months of September 2019 to January 2020.100 children who are diagnosed with severe pneumonia were included in this the study.

Results: A total of 100 children have been enrolled in the present study. Among them $56(56 \%)$ are Males and $44(44 \%)$ are Females. Male: female ratio was 1.3: 0.8. Treatment Failure rate in oral amoxicillin group is $24 \%$ and in Inj.ampicillin plus Amikacin (parenteral) group is $16 \%$. The difference in treatment outcome in the two groups is not statistically significant. $(p=1.0000, p=0.3173)$.

Conclusion: Proper training of care givers and treating clinicians to promptly recognize the danger signs of very severe pneumonia, one can safely treat the patients with severe pneumonia with oral Amoxicillin.
\end{abstract}

Keywords: Pneumonia, oral amoxicillin, parenteral ampicillin, amikacin

\section{Introduction}

Pneumonia is an acute inflammation of the lung parenchyma that can be due to infective and non-infective aetiology, the clinical presentation with physical and radiological features are compatible with pulmonary consolidation of one or both lungs ${ }^{[1]}$. The most significant and striking feature of pneumonia is consolidation. Pneumonia continues to be the biggest killer disease globally, of less than five year's children. In India, pneumonia caused nearly 175,000 child deaths in $2013^{[2]}$. Pneumonia accounts for $15 \%$ of all deaths of children under 5 years old, killing 8, 08694 children in 2017. Data shows that the majority of childhood pneumonia deaths are due to severe pneumonia ${ }^{[3]}$. Management of these severe pneumonia cases requires early identification, referral and the provision of good-quality higher- level care. However, in many low-resource settings, referral is difficult and often does not take place ${ }^{\text {[4- }}$ 7]. According to national family health survey 2014-15 (NFHS 4), under- five mortality rate in Telangana is 32 per 1000 live birth ${ }^{[8]}$. Community acquired pneumonia being a major contributor to the under-five mortality in the state of Telangana. Aim and Objective of this study is to compare the effectiveness of oral versus parenteral antibiotics in the management of severe pneumonia in children under five years of age.

\section{Methodology}

Hospital based prospective observational comparative study. This study was conducted in the department of paediatrics, RVM institute of medical sciences and research canter. This study was approved by the RVM institutional ethical committee prior to the study. The study was conducted between the months of September 2019 to January 2020.100 children were included in this study. Informed consent was taken from the parents/attendants of the study participants.

Inclusion Criteria: Children from 6 months to 59 months old who are diagnosed as severe pneumonia. 
Exclusion Criteria: Children with co-morbidities such as HIV/AIDS Congenital heart diseases, Tuberculosis. Parents/Attendants who are not willing to participate in the study. Detailed clinical examination with relevant investigations like chest X-ray, complete blood picture, blood culture was also analysed. Children were allotted to either "oral group" or "parenteral group". Children in the parenteral group received intravenous Ampicillin at $100 \mathrm{mg} / \mathrm{kg}$ 6th hourly plus Amikacin at $15 \mathrm{mg} / \mathrm{kg} 12$ th hourly. Children in the oral group received, oral amoxicillin, dose of $40-45 \mathrm{mg} / \mathrm{kg}$ 8th hourly. Observation time was minimum 3 days or till discharge. Appropriate statistical tests were applied using software SPSS version 20. Percentages, $\mathrm{p}$ values using chi square test was calculated wherever necessary.

\section{Results}

Table 1: Showing the gender and age distribution

\begin{tabular}{|c|c|c|c|c|}
\hline Age(months) & Male & Female & Total & Percentage \\
\hline 6 to 12 & 6 & 4 & 10 & 10 \\
\hline 13 to 24 & 10 & 6 & 16 & 16 \\
\hline 25 to 36 & 14 & 8 & 22 & 22 \\
\hline 37 to 48 & 11 & 9 & 20 & 20 \\
\hline 49 to 59 & 15 & 17 & 32 & 32 \\
\hline Total & 56 & 44 & 100 & 100 \\
\hline
\end{tabular}

A total of 100 children have been enrolled in the present study. Among them 56 (56\%) are Males and 44 (44\%) are Females. In this study majority $(n=32 ; 32 \%)$ of the children are between 49-59 months. This is nearly one third of the total study population

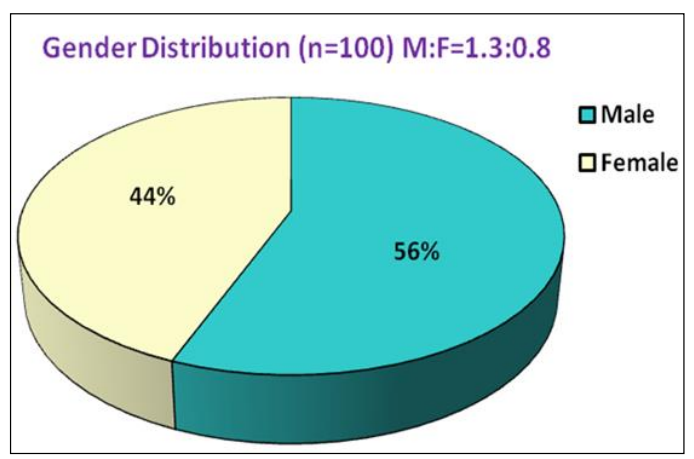

Fig 1: Gender distribution among study participants

The predominant participants are males with, Male: female ratio was 1.3: 0.8 in this study.

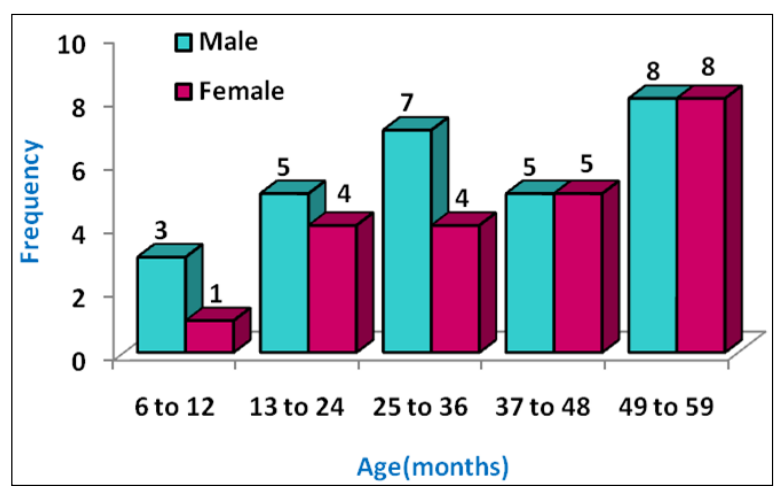

Fig 2: Age \& Sex wise distribution in oral Amoxicillin group among study participants
A total of 50 children were included in the amoxicillin group. Of them $32 \%(n=16)$ are in the age group between 49 months to 59 months. Children between age group 25 to 36 months constitute $22 \%(n=11)$ Children between 37 to 48 months constitute $20 \%(n=10)$

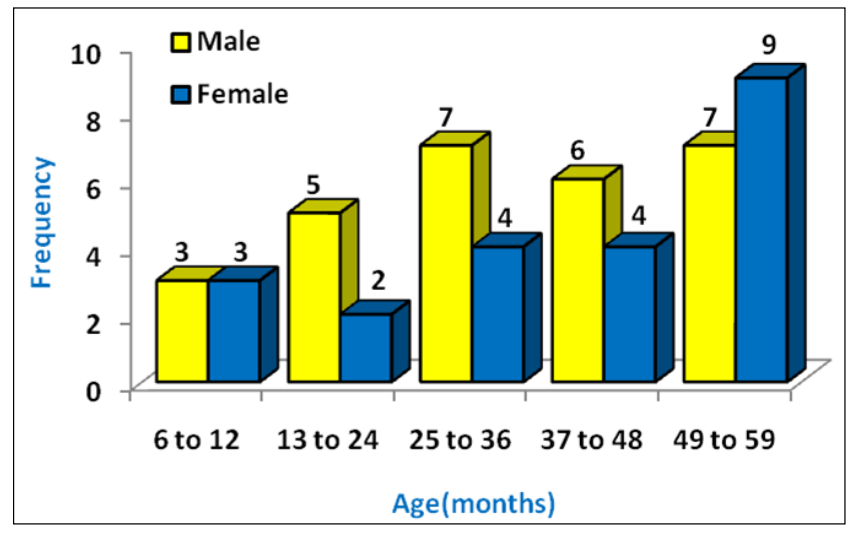

Fig 3: Gender and age distribution in parenteral treatment group

A total of 50 children were included in the Inj.ampicillin plus Inj.amikacin group. Majority of them belong to the age group of 49 to 59 months (32\%), followed by the age group 25 to 36 months $(22 \%) .37$ to 48 months constitute $(20 \%)$, 13 to 24 months constitute $14 \%, 6$ to 12 months constitute $12 \%$.

Table 2: Distribution of acute malnutrition among the oral and parenteral groups

\begin{tabular}{|c|c|c|c|c|c|}
\hline \multirow{3}{*}{ Status of Nutrition } & \multicolumn{4}{|c|}{ Type of treatment received } & \multirow{2}{*}{$(\chi 2, P<0.05) *$} \\
\hline & \multicolumn{2}{|c|}{ Oral } & \multicolumn{2}{|c|}{ Parenteral } & \\
\hline & No & $\%$ & No & $\%$ & \multirow{4}{*}{$(0.1623,0.6870)$} \\
\hline Acute malnutrition & 21 & 42 & 23 & 46 & \\
\hline Normal & 29 & 58 & 27 & 54 & \\
\hline Total & 50 & 100 & 50 & 100 & \\
\hline
\end{tabular}

$*_{-} P<0.05$ there is no significant association between Treatment group and Status of Nutrition by using chi-square test

Among the 50 study participants in the oral amoxicillin group $21(42 \%)$ children had Acute Malnutrition, while among the 50 in the parenteral group, 23 (46\%) children had Acute Malnutrition. There is no statistically significant difference in the distribution of the children with moderate acute malnutrition among the two study populations $(\mathrm{p}=0.1623, \mathrm{p}=0.6870)$.

Table 3: Distribution of study participants with anaemia among the oral and parenteral groups

\begin{tabular}{|c|c|c|c|c|c|}
\hline \multirow{3}{*}{ Anaemia } & \multicolumn{4}{|c|}{ Treatment group } & \multirow{2}{*}{$(\chi 2, P<0.05) *$} \\
\hline & \multicolumn{2}{|c|}{ Oral } & \multicolumn{2}{|c|}{ Parenteral } & \\
\hline & No & $\%$ & No & $\%$ & \multirow{4}{*}{$(0.7352,0.3912)$} \\
\hline Anaemic children & 18 & 36 & 14 & 28 & \\
\hline Normal children & 32 & 64 & 36 & 72 & \\
\hline Total & 50 & 100 & 50 & 100 & \\
\hline
\end{tabular}

$*_{-} P<0.05$ there is no significant association between Treatment group and Status of Anemia by using chi-square test

Among the 50 study participants in the oral amoxicillin group $18(36 \%)$ children had anaemia, while among the 50 study participants in the parenteral group, $14(28 \%)$ children had anemia. There is no statistically significant difference in the distribution of the children with anaemia among the two study populations $(\mathrm{p}=0.7352, \mathrm{p}=0.3912)$ 


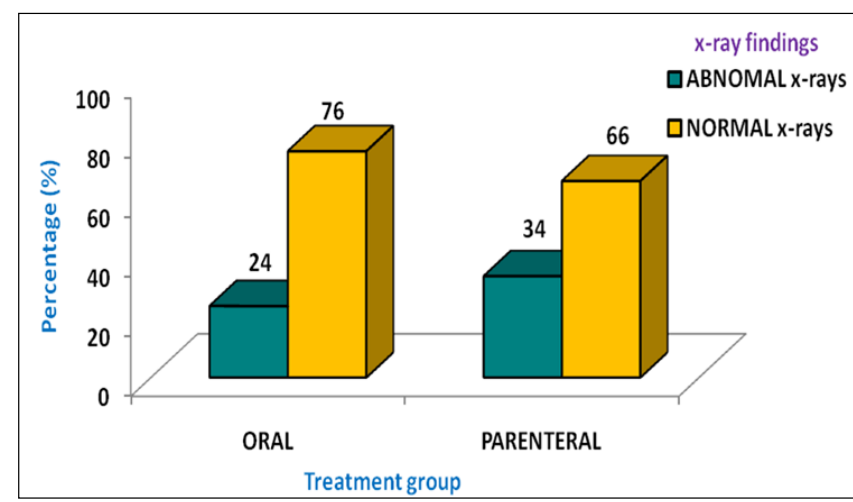

Fig 4: Showing the normal and abnormal chest $X$ rays in oral and parenteral group

Among the 50 study participants in the oral amoxicillin group, chest $\mathrm{x}$ ray was done in all 50 children. Out of the 50 children only $24 \%(n=12)$ children had abnormal chest $\mathrm{x}$ ray findings, while the other $76 \%(n=38)$ children had normal chest $X$ ray findings and Among the 50 study participants in the parenteral group, chest $\mathrm{X}$ ray was done in all 50 children. Of this 50 children only $34 \% \quad(n=17)$ children had abnormal chest $\mathrm{x}$ ray findings, while the other $66 \%(n=33)$ children had normal chest $x$ ray findings.

Table 4: Treatment outcome in oral amoxicillin group versus Inj.Ampicillin plus Amikacin (parenteral) group

\begin{tabular}{|c|c|c|c|c|c|}
\hline \multirow{3}{*}{ Treatment group } & \multicolumn{4}{|c|}{ Treatment outcome } & \multirow{2}{*}{$(\chi 2, P<0.05) *$} \\
\hline & \multicolumn{2}{|c|}{ Oral } & \multicolumn{2}{|c|}{ Parenteral } & \\
\hline & No & $\%$ & No & $\%$ & \multirow{4}{*}{$(1.0000,0.3173)$} \\
\hline Success & 38 & 76 & 42 & 84 & \\
\hline Failure & 12 & 24 & 8 & 16 & \\
\hline Total & 50 & 100 & 50 & 100 & \\
\hline
\end{tabular}

*- $P<0.05$ there is no significant association between Treatment group and Treatment outcome by using chi-square test

Treatment Failure rate in oral amoxicillin group is $24 \%$ and in Inj.ampicillin plus Amikacin (parenteral) group is $16 \%$. The difference in treatment outcome in the two groups is not statistically significant. $(p=1.0000, p=0.3173)$

\section{Discussion}

In the present study a total of 100 children between 6 months to 59 months have been selected. Of the total children, 50 received oral amoxicillin and 50 received I.V Ampicillin plus amikacin. The study populations in these studies conducted by Ambrose et al. ${ }^{[9]}$, hazir et al. ${ }^{[10]}$ and Addo yobo et al. ${ }^{[11]}$ are comparatively large because they are multi centric trials, whereas this study population is confined to a single center. In a study conducted by Ambrose et al, males were $57.1 \%$ and females were $42.8 \%$. In Hazir et al. ${ }^{[10]}$ study, males were $60.4 \%$ and females were $39.5 \%$. In the present study males were $56 \%$ and females were $44 \%$ these findings are similar with the above studies where male children are more than female children. The results of the current study are consistent with other large multi-centre trials ${ }^{[41-43]}$. Treatment failure rates in the different studies have varied from $7.5 \%$ to $19 \%$ in the oral amoxicillin group and from $8.6 \%$ to $19 \%$ in the Inj.penicillin group. This variation in the failure rates can be attributed to the wide variation in the study population, study setting. In the open-label, multicentre randomized controlled non-inferiority trial conducted by Ambrose agweyu et al. ${ }^{[9]}$ treatment failure by day 5 post enrolment was $11.4 \%$ and $11.0 \%$ in the amoxicillin and benzyl penicillin groups respectively. In the randomised, open-label equivalency trial at seven study sites in Pakistan by Hazir et al. ${ }^{[12]}$, there were $87(8.6 \%)$ treatment failures in the hospitalised group and $77(7.5 \%)$ in the ambulatory group (risk difference $1.1 \%$; $95 \% \mathrm{CI}-1.3$ to 3.5 ) by day 6 . The multicentre, randomised, open-label equivalency study undertaken at tertiary-care centers in eight developing countries in Africa, Asia, and South America by Addo yobo et al. ${ }^{[13]}$ (APPIS study), treatment failure was $19 \%$ in each group (161 patients in penicillin arm; 167 patients in amoxicillin arm; risk difference $-0.4 \% ; 95 \% \mathrm{CI}-4 \cdot 2$ to $3 \cdot 3$ ) at $48 \mathrm{hr}$. In the Present study, treatment failure in oral Amoxicillin group is $24 \%$ and in the Inj.Ampicillin+Amikacin group is $16 \%$ by day 5. The difference in the failure rates in the oral and parenteral groups of the present study is statistically not significant. The treatment failure rates of the present study are comparable with the above studies.

\section{Conclusion}

In the present study it has been observed that there is no statistically significant difference in the failure rate in oral amoxicillin group and Inj.Ampicillin plus Inj.Amikacin group, suggesting similar outcome for severe pneumonia treated with oral amoxicillin and Inj.Ampicillin plus Amikacin. From the results of the present study it can be concluded that by careful selection of study participants by excluding those with potential comorbidities like malnutrition, immunodeficiency, etc and by proper training of care givers and treating clinicians to promptly recognize the danger signs of very severe pneumonia, one can safely treat the patients with severe pneumonia with oral Amoxicillin.

\section{References}

1. Seaton A, Seaton D, Leich AG, Crofton, Douglas's. Respiratory Diseases. 5th edition. 1(13):356-429.

2. Shann F. Etiology of severe pneumonia in children in developing countries. Pediatr Infect Dis J. 1986; 5:24752.

3. Rudan I et al. Epidemiology and etiology of childhood pneumonia. Bulletin of the World Health Organization. 2008; 86:408-416.

4. Bang AT, Bang RA, Reddy HM. Home-based neonatal care: summary and applications of the field trial in rural Gadchiroli, India (1993 to 2003). Journal of Perinatology. 2005; 25:S108-S122.

5. Aguilar AM et al. Mortality Survey of Bolivia: The Final Report: Investigating and identifying the causes of death for children under five. Published for USAID by the Basic Support for Institutionalizing Child Survival (BASICS) Project, Virginia, 1998.

6. Schumacher $\mathrm{R}$ et al. Mortality Study in Guinea: Investigating the causes of death in children under 5 . Published by Save the Children and the Basic Support for Institutionalizing Child Survival (BASICS II) Project, Virginia, 2002.

7. Schellenberg JA et al. Inequalities among the very poor. Health care for children in rural southern Tanzania. Lancet. 2003; 361:561-66.

8. National family health survey 4 (NFHS 4), Ministry of health and family welfare, Government of India.

9. Ambrose Agweyu, Davis Gathara et al Oral 
Amoxicillin Versus Benzyl Penicillin for Severe Pneumonia Among Kenyan Children Pragmatic Randomized Controlled Noninferiority Trial.

10. Hazir T, Fox LM, Bin Nisar YB, Fox MP, Ashraf YP et al. Ambulatory short-course high-dose oral amoxicillin for treatment of severe pneumonia in children: a randomised equivalency trial. Lancet. 2008; 371:49-56.

11. Addo-Yobo E, Chisaka N, Hassan M, Hibberd P, Lozano JM et al. Oral amoxicillin versus injectable penicillin for severe pneumonia in children aged 3 to 59 months: a randomised multicentre equivalency study. Lancet. 2004; 364:1141-1148.

12. Hazir T, Fox LM, Nisar YB et al. Ambulatory shortcourse high-dose oral amoxicillin for treatment of severe pneumonia in children: a randomized equivalency trial. Lancet. 2008; 371:49-56.

13. Addo-Yobo E, Chisaka N, Hassan $M$ et al. Oral amoxicillin versus injectable penicillin for severe pneumonia in children aged 3 to 59 months: a randomised multicentre equivalency study. Lancet $2004 ; 364: 1141-8$. 